\title{
AN INVERTED LIST BASED APPROACH TO GENERATE OPTIMISED PATH IN DSR IN MANETS
}

\author{
Sunita \\ Department of Computer \\ Science and Engineering. \\ BPSMV, Khanpur Kalan \\ Sonepat,India
}

\author{
Kusum Lata \\ Department of Computer \\ Science and Engineering \\ BPSMV, Khanpur Kalan, \\ Sonepat,India
}

\author{
Sophia Dhankhar \\ Department of Computer \\ Science and Applications \\ DCSA Department \\ Rohtak,India
}

\begin{abstract}
In this paper, we design and formulate the inverted list based approach for providing safer path and effective communication in DSR protocol.Some nodes in network can participate in network more frequenctly whereas some nodes are not participating. Because of this there is the requirement of such an approach that will take an intelligent decision regarding the sharing of bandwidth or the resource to a node or the node group.Dynamic source routing protocol (DSR) is an on-demand, source routing protocol, whereby all the routing information is maintained (continually updated) at mobile nodes.DSR uses a reactive approach which eliminates the need to periodically flood the network with table update messages which are required in a table-driven approach. The intermediate nodes also utilize the route cache information efficiently to reduce the control overhead.
\end{abstract}

Keywords:MANET,Ad hoc, DSR, Routing Algorithm, Reverse Route

\section{INTRODUCTION}

A Mobile ad hoc network is a group of wireless mobile computers (or nodes). In which nodes collaborate by forwarding packets for each other to allow them to communicate outside range of direct wireless transmission. Ad hoc networks require no centralized administration or fixed network infrastructure such as base stations or access points, and can be quickly and inexpensively set up as needed.A MANET is an autonomous group of mobile users that communicate over reasonably slow wireless links. The network topology may vary rapidly and unpredictably over time, because the nodes are mobile. The network is decentralized, where all network activity, including discovering the topology and delivering messages must be executed by the nodes themselves. Hence routing functionality will have to be incorporated into the mobile nodes.[1,2]

MANET is a kind of wireless ad-hoc network and it is a selfconfiguring network of mobile routers (and associated hosts) connected by wireless links - the union of which forms an arbitrary topology. The routers, the participating nodes act as router, are free to move randomly and manage themselves arbitrarily; thus, the network's wireless topology may change rapidly and unpredictably. Such a network may operate in a standalone fashion, or may be connected to the larger Internet Problems in Ad Hoc Networks.[7]

Dynamic Source Routing (DSR) is a routing protocol for wireless mesh networks. It is similar to in that it establishes a route on-demand when a transmitting mobile node requests one. AODV However, it uses source routing instead of relying on the routing table at each intermediate device. Dynamic source routing protocol (DSR) is an on-demand, source routing protocol ,whereby all the routing information is maintained (continually updated) at mobile nodes. DSR allows the network to be completely self-organizing and selfconfiguring, without the need for any existing network infrastructure or administration. The protocol is composed of the two main mechanisms of "Route Discovery" and "Route
Maintenance", which work together to allow nodes to discover and maintain routes to arbitrary destinations in the ad hoc network

An optimum path for a communication between a source node and target node is determined by Route Discovery process. Route Maintenance ensures that the communication path remains optimum and loop-free according the change in network conditions, even if this requires altering the route during a transmission. Route Reply would only be generated if the message has reached the projected destination node (route record which is firstly contained in Route Request would be inserted into the Route Reply). $[3,4]$

The objective of this paper is Implementation of Wireless Network in the Real Environment. Design an algorithm using inverted list approach to identify the selfish node and to perform the communication effectively.Implementation of proposed system on to the network under DSR prootcol.Analyze the work over the network. [5]

The remaining paper is described as section 2 describes the work related to DSR the proposed scheme is described in section3.Section4 represents the experimental results.Section5 represents the conclusion and then references.[6]

\section{RELATED WORK}

Debdutta Barman Roy propose a new Intrusion Detection System (IDS) based on Mobile Agents. The approach uses a set of Mobile Agent (MA) that can move from one node to another node within a network. This as a whole reduces network bandwidth consumption by moving the computation for data analysis to the location of the intrusion. Besides, it has been established that the proposed method also decreases the computation overhead in each node in the network. [8] 
Shailender Gupta defined a work on selfish node detection. A selfish node is one that tries to utilize the network resources for its own profit but is reluctant to spend its own for others. If such behaviour prevails among large number of the nodes in the network, it may eventually lead to disruption of network. This paper studies the impact of selfish nodes concentration on the quality of service in MANETs. [9]

Md. Amir Khusru Akhtar presented a mathematical model to detect the selfish node. In this paper Author are presenting the mathematical model to detect selfish nodes using the probability density function. The proposed model works with existing routing protocol and the nodes that are suspected of having the selfishness are given a Selfishness test. This model formulates this problem with the help of prior probability and continuous Bayes' theorem.[10]

Li Zhao performed a work to detect misbehaviour on data and mitigate adverse effects, Author propose and evaluate a Multipath Routing Single path transmission (MARS) scheme. The MARS combines multipath routing, single path data transmission, and end-to-end feedback mechanism together to provide more comprehensive protection against misbehaviour from individual or cooperating misbehaving nodes. [11]

Zougagh Hicham performed a comparative study of intrusion detection in adhoc nework. In recent years, the use of mobile ad hoc network (MANETs) has been widespread in many applications. Due to its deployment nature, MANETs are more vulnerable to malicious attack. The absolute security in the mobile ad hoc network is very hard to achieve because of its fundamental characteristics, such as dynamic topology, open medium, absence of infrastructure, limited power and limited bandwidth. In this article Author classify the architecture for IDS that have so far been introduced for MANETs, and then existing intrusion detection techniques in MANETs presented and compared. Author then provide some directions for future researches. [12]

Michael Wayne Probus performed a work on selfish node isolation. This thesis will focus on the topic of Selfish Nodes within a Mobile Ad-Hoc Networks (MANET), specifically sensor networks due to their lower power and bandwidth. The approach used is a reputation based algorithm to isolate the selfish nodes from communication by using past history to determine how reliable the node is. The reputation of each node is determined by their behavior within the network. As a node continuously acts selfishly, their reputation is decreased, until finally meeting the minimum threshold; therefore they are determined to be malicious. $[13,14,15]$

\section{PROPOSED WORK}

\subsection{Significance of Work}

In this work presented work a to find the frequent communicating nodes over the network The proposed system can be implemented on a wired or wireless network. The proposed system is also independent to the network type. It can be mobile network or the wireless lan. . It is the intelligent system that uses the artificial intelligent system approach along with statistical analysis to derive the fair and quick results about the study of allocation of resources to the available nodes

\subsection{Research Design}

The proposed work is about to find the most frequent moving pattern over the network so that we can find the nodes or the nodes pair that should get the maximum concern respective to the resource allocation.It means the node participating regularly over the network should get more resources. Here the term resource defines the time slice or the bandwidth of the network.

The complete Research Design is given as

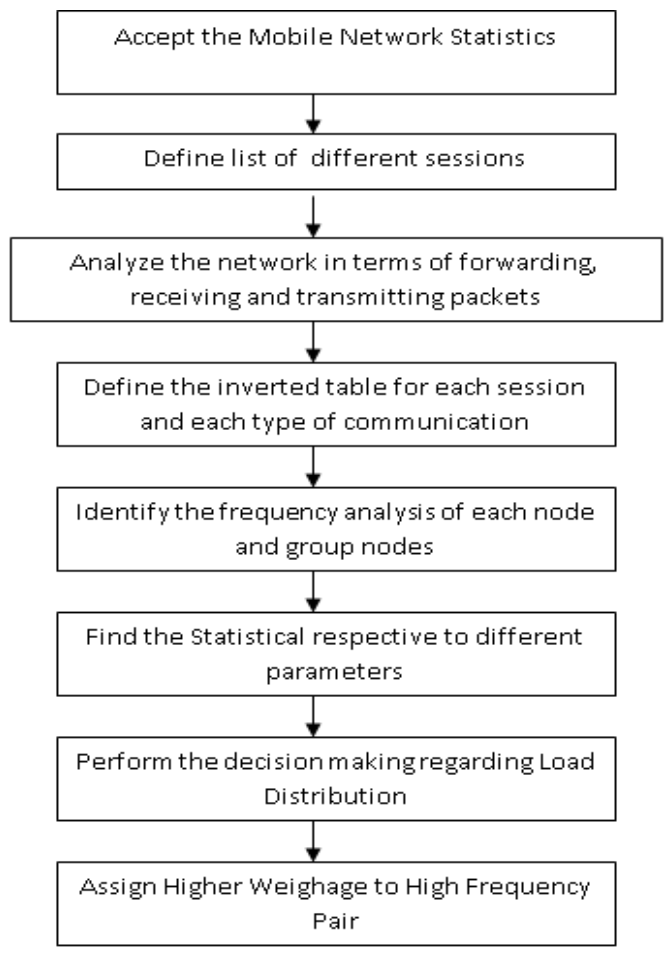

In this work the main concern is about to find the frequency of node participation over the network. Lot of work is done in the same direction. Here we are presenting the improved inverted table mechanism to find the most frequent nodes over the network. The method is introduced by K.V.S.R.P.Varma in year 2010. This approach is used by him to idenfiy the similarity and frequency analysis in case of DNA sequencing. $\mathrm{He}$ performed the work to find the largest possible node sequence over the network.

Lot of work is already done in terms of string extraction, string matching and pattern identification over the string. Frequent Item set Mining plays an essential role in many data mining tasks and applications, such as mining association rules, correlations, sequential patterns, classification and clustering. Frequent item set construction has been a major research area over the years and several algorithms have been proposed in the literature to address the problem of mining association rules.

\section{SIMULATION AND RESULTS}

\subsection{Simulator Study- The Network Simulator (NS2)}

In simulation, we can construct a mathematical model to reproduce the characteristics of a phenomenon, system, or process often using a computer in order to information or solve problems. Nowadays, there are many network simulators that can simulate the MANET. In this section we will introduce the most commonly used simulators. We will 
compare their advantages and disadvantages and choose one to as platform to implement reactive/proactive protocol and conduct simulations in this thesis.

\subsubsection{NS2 Overview}

Ns-2 is a discrete event simulator targeted at networking research. It provides substantial ssupport for simulation of $\mathrm{TCP}$, routing and multicast protocols over wired and wireless networks. It consists of two simulation tools. The network simulator (ns) contains all commonly used IP protocols. The network animator (nam) is use to visualize the simulations. Ns-2 fully simulates a layered network from the physical radio transmission channel to high-level applications.

Version 2 is the most recent version of ns (ns-2). The simulator was originally developed by the University of California at Berkeley and VINT project the simulator was recently extended to provide simulation support for ad hoc network by Carnegie Mellon University (CMU Monarch Project homepage, 1999).

Ns-2 is an object-oriented simulator written in $\mathrm{C}++$ and OTcl. The simulator supports aclass hierarchy in $\mathrm{C}++$ and a similar class hierarchy within the OTcl interpreter. There is a one-toone correspondence between a class in the interpreted hierarchy and one in thecompile hierarchy. The reason to use two different programming languages is that OTclis suitable for the programs and configurations that demand frequent and fast change while $\mathrm{C}++$ is suitable for the programs that have high demand in speed. Ns-2 is highly extensible. It not only supports most commonly used IP protocols but also allows the users to extend or implement their own protocols. It also provides powerful trace functionalities, which are very important in our project since various information need to be logged for analysis. The full source code of ns-2 can be downloaded and compile for multiple platforms such as UNIX, Windows and Ubuntu.

\subsection{Analysis Results}

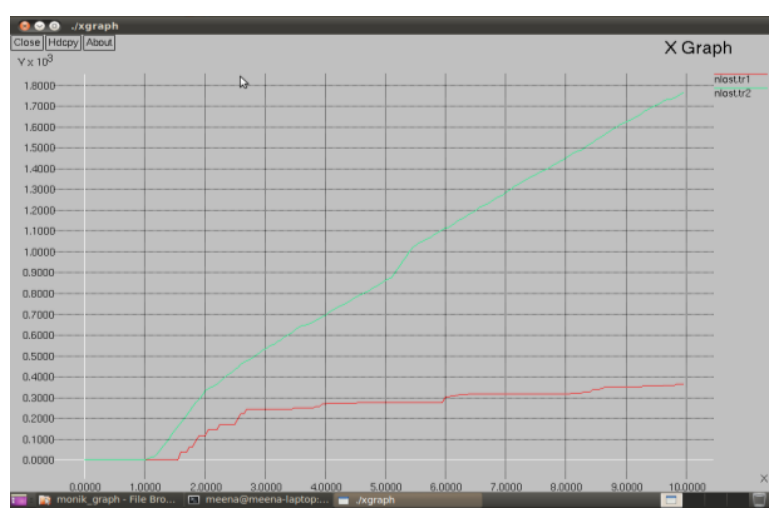

Figure 4.1:Packet Lost (Existing Vs Proposed Approach)

Here figure 4.1 is showing the comparative analysis of packet lost over the network. Here $\mathrm{x}$ axis represents the time and $\mathrm{y}$ axis represents the packet transmitted. As we can see after implementing the proposed approach the packet loss over the network is decreased.

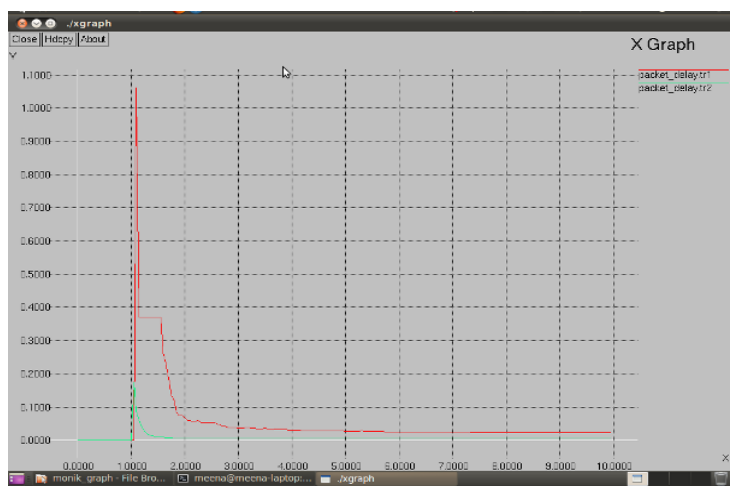

Figure 4.2 : Packet Delay (Existing Vs Proposed Approach)

Here figure 4.2 is showing the comparative analysis of Packet Delay over the network. Here $\mathrm{x}$ axis represents the time and $\mathrm{y}$ axis represents the Packet Delay of communication. As we can see after implementing the proposed approach the Packe $t$ Delay over the network is decreased.

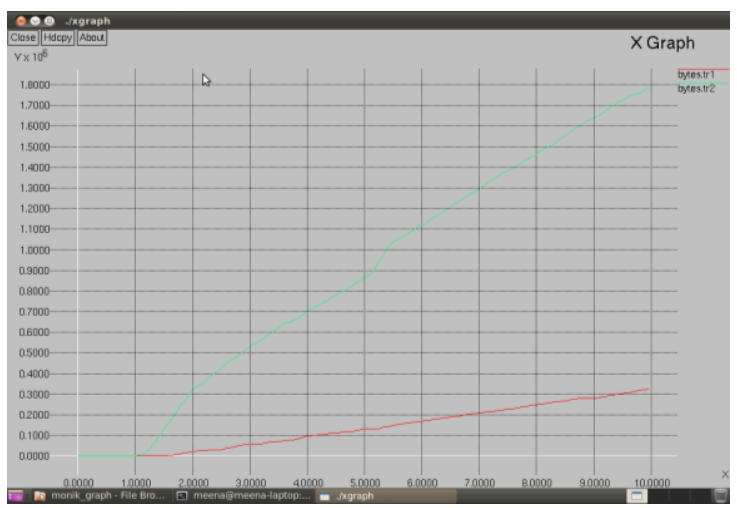

Figure 4.3 :Bytes transmitted (Existing Vs Proposed Approach)

Here figure 4.3 is showing the comparative analysis of bytes transmitted over thenetwork. Here $\mathrm{x}$ axis represents the time and $y$ axis represents the btes transmitted. As we can see after implementing the proposed approach the bytes transmitted over the network is increased.

\section{CONCLUSION}

The proposed work is about the prevention of Selfish Node attack. The proposed work is about to improve the DSR protocol in terms of security. As in case of multicast network because of lot of communication the network suffer from some attack that results the packet loss over the network. The proposed work is about to minimize this packet loss over the network. The work will increase the throughput with this 
improved DSR protocol. The system is providing better throughput and less packet loss over the network. The system is implemented in a wireless network with DSR protocol. In this system an improved inverted list approach is defined to perform the analysis among neighboring nodes and to provide the communication from effective path. Here we have proposed a new algorithm for the above said task. The implementation is performed in $\mathrm{ns} 2$ and analysis is presented using xgraph.

\section{REFERENCES}

[1] Debdutta Barman Roy," MADSN: Mobile Agent Based Detection of Selfish Node in MANET", International Journal of Wireless \& Mobile Networks (IJWMN) Vol. 3, No. 4, August 2011

[2] Shailender Gupta," IMPACT OF SELFISH NODE CONCENTRATION IN MANETS", International Journal of Wireless \& Mobile Networks (IJWMN), ISSN : 0975-3834 [Online] ; 0975-4679, Volume: 3 - volume NO: 2 - Issue: April 2011

[3] DipaliKoshti," Comparative study of Techniques used for Detection of Selfish Nodes in Mobile Ad hoc Networks", International Journal of Soft Computing and Engineering (IJSCE) ISSN: 2231-2307, 2011

[4] S.Usha," Multi Hop Acknowledgement Scheme based Selfish Node Detection in Mobile Ad hoc Networks", International Journal of Computer and Electrical Engineering, International Journal of Computer and Electrical Engineering, Vol. 3, No. 4, August 2011

[5]Martin Schütte," Detecting Selfish and Malicious Nodes in MANETs", SEMINAR: SICHERHEIT IN SELBSTORGANISIERENDEN NETZEN, HPI/UNIVERSITÄT POTSDAM, SOMMERSEMESTER 2006

[6]Li Zhao," MARS: Misbehavior Detection in Ad Hoc Networks", Global Telecommunications Conference, 2007. GLOBECOM '07. IEEE, 26-30 Nov. 2007, 941 945

[7] Md. Amir KhusruAkhtar," Mathematical Model for the Detection of Selfish Nodes in MANETs", International Journal of Computer Science and Informatics (IJCSI) ISSN (PRINT): 2231 -5292, Volume-1, Issue-3

[8] KhairulAzmi Abu Bakar," Contribution Time-based Selfish Nodes Detection Scheme".

[9] Hongxun Liu," USING A CACHE SCHEME TO DETECT SELFISH NODES IN MOBILE AD HOC NETWORKS", Proceeding CIIT '07 The Sixth IASTED International Conference on Communications, Internet, and Information Technology, ACTA Press Anaheim, CA, USA @2007

[10] Rekhakaushik," DETECTION AND ISOLATION OF RELUCTANT NODES USING REPUTATION BASED SCHEME IN AN AD-HOC NETWORK",International Journal of Computer Networks \& Communications (IJCNC), Vol.3, No.2, March 2011.

[11] Frank Kargl," Advanced Detection of Selfish or Malicious Nodes in Ad hoc Networks", Proceeding ESAS'04 Proceedings of the First European conference on Security in Ad-hoc and Sensor Networks Pages 152165, ISBN:3-540-24396-8, 2005

[12] Jamal N. Al-Karaki,"Stimulating Node Cooperation in Mobile Ad hoc Networks Wireless. PersCommun (2008) 44:219-239

[13] Bo Wang," Local Detection of Selfish Routing Behavior in Ad Hoc Networks", ISPAN '05 Proceedings of the 8th International Symposium on Parallel Architectures,Algorithms and Networks Pages 392 - 399

[14] Deepak Kumar Dixit," A Trust Based Scheme to Encourage Packet Forwarding in Mobile Ad-hoc Networks", (IJCSIT) International Journal of Computer Science and Information Technologies, Vol. 3 (3), 2012,4327 - 4330, ISSN:0975-9646

[15] Anuj Joshi," Efficient Content Authentication in Ad-Hoc Networks- Mitigating DDoS Attacks", International Journal of Computer Applications (0975 - 8887) 